\title{
Planning the Expansion of Long-Term Transmission Networks Using a Cycle-Based Formulation
}

\author{
Pedro Pablo Cardenas Alzate ${ }^{1}$, Laura Monica Escobar Vargas ${ }^{2}$ \& Antonio Hernando Escobar Zuluaga ${ }^{3}$ \\ ${ }^{1}$ Department of Mathematics, Universidad Tecnologica de Pereira, Colombia \\ ${ }^{2}$ Department of electrical engineering, Sao Paulo State University UNESP, Ilha Solteira, SP, Brazil \\ ${ }^{3}$ School of Electrical Technology, Universidad Tecnol'ogica de Pereira, Colombia \\ Correspondence: Pedro Pablo Cardenas Alzate, Department of Mathematics, Universidad Tecnologica de Pereira, \\ Colombia. E-mail: ppablo@utp.edu.co
}

Received: November 7, 2018

doi:10.5539/mas.v13n1p237
Accepted: Nov. 16, $2018 \quad$ Online Published: December 31, 2018

URL: https://doi.org/10.5539/mas.v13n1p237

\begin{abstract}
This paper presents a methodology to solve the long-term transmission expansion planning problem, using a formulation that uses mathematical expressions that are alternatives to the second Kirchhoff's law and that are applied to the cycles critical of the system graph. The network transmission expansion planning problem of power systems is part of the socalled NP-complete problems, which belong to a category of problems that are dfficult to solve, for which polynomial solution algorithms are not known. The proposed methodology is applied to two test systems of the specialized literature with very good results.
\end{abstract}

Keywords: optimization, transmission planning, critical cycles, Kirchhoff's second law

\section{Introduction}

The problem of planning the expansion of the electricity transmission network determines the new investments in electrical infrastructure: transmission lines and substations, required to allow the adequate transfer of active power between the generation centers and the energy consumption centers, in a planning horizon of 10 or more years (Escobar et al, 2010).

The investment options associated with this problem are characterized by high costs, by the long periods of time required for its construction, by the long periods of recovery of the investment and by the need to keep the built infrastructure in operation until such investment is recovered. This problem takes as a reference the current network and considers the future increase of the demand in the nodes of the system, the existing generation, the alternatives of new generation, the repowering of the existing generation, the new demand nodes, the requirements of new substations and the repowering of existing substations, in the analyzed time horizon.

Static planning determines the minimum cost solution considering a single period of time in the planning horizon, this is denominated static planning, and assumes that the existing network is part of the optimal future solution, which means that the possibility of withdrawing, moving or leaving elements that are operating in the current network, is not considered. This is a problem of optimization of integer-mixed nonlinear programming (IMNLP). It is a multimodal, non-convex problem that can not be solved successfully using exact optimization techniques when the system is large and has a large number of isolated nodes. In systems of smaller dimensions, the optimal solution is found using methods such as Branch and Cut or Branch and Bound (Escobar et al, 2010; Dom' inguez, 2014). In some of these cases, it is found that computer systems require large calculation times compared to those required by metaheuristic techniques such as (Tabu Search (TS) or the genetic algorithm proposed by Chu and Beasley (CBGA) (Escobar et al, 2016), which do not guarantee obtaining the optimal global solution. The problems that are simultaneously IMNLP and NP-complete are characterized as being the most difficult solution. To solve the problem of expansion planning, various methodologies have been used from heuristic and metaheuristic techniques, to exact methods (Dominguez, 2014).

In this paper a novel formulation of the original problem is proposed, replacing the Kirchhoff's second law by restrictions associated with the critical cycles of the system, which produce the same optimal solution with less computational effort (Escobar L, 2018). The problem is solved initially using the transport model. Then, the 
existing circuits at their upper limit and the investment in new corridors are identified in the relaxed solution. These corridors are identified and included in a cycle restriction or closed trajectory that must meet two conditions: it must contain the identified circuit and must associate this circuits with the paths that form a cycle with the least amount of products power-reactance, in which the relative direction of the active power flows is taken into account. The identified cycles are added to the transport model, and the problem is solved again, repeating the previous process until no overloaded circuits or investment in new corridors appear in the solution. This procedure allows to identify critical cycles, and allows to find the optimal solution obtained with the DC model, for this problem, without including Kirchhoff's second law in the traditional way.

\section{Method}

\subsection{Problem Formulation}

The mathematical model used to solve the problem of transmission planning is the following:

$$
\begin{array}{ll}
\text { min } & =\sum_{(i, j) \in \Omega_{1}} C_{i j} \sum_{k \in \Omega_{2}} y_{i j, k} \\
\text { s.a. } & \sum_{(p, i) \in \Omega_{1}}\left(f_{p i}^{0}+\sum_{k \in \Omega_{2}} f_{p i, k}\right)-\sum_{(i, j) \in \Omega_{1}}\left(f_{i j}^{0}+\sum_{k \in \Omega_{2}} f_{i j, k}\right)+g_{i}=d_{i} \\
& \left|f_{i j}^{0}\right| \leq n_{i j}^{0} \bar{f}_{i j}, \quad \forall(i, j) \in \Omega_{1} \\
& \left|f_{i j, k}\right| \leq y_{i j, k} \bar{f}_{i j}, \quad \forall(i, j) \in \Omega_{1}, k \in \Omega_{2} \\
& 0 \leq g_{i} \leq \bar{g}_{i} \\
& \sum_{k \in \Omega_{2}} y_{i j, k} \leq \bar{n}_{i j}, \quad \forall(i, j) \in \Omega_{1} \\
& y_{i j, k-1} \geq y_{i j, k}, \quad \forall(i, j) \in \Omega_{1}, k \in \Omega_{2}, k>1 \\
& \left|f_{i j, k}-f_{i j, k-1}\right| \leq M\left(1-y_{i j, k}\right), \quad \forall(i, j) \in \Omega_{1}, k \in \Omega_{2}, k>1 \\
& \left|\left(f_{i j}^{0} / n_{i j}^{0}-f_{i j, 1}\right)\right| \leq M\left(1-y_{i j, 1}\right), \quad \forall(i, j) \in \Omega_{1}, k \in \Omega_{2}, n_{i j}^{0}>0
\end{array}
$$

In the previous model, $C_{i j}$ is the cost of adding a circuit in the $i j$ corridor. $\Omega_{1}$ is the set of existing and expansion corridors. $y_{i j, k}$ is the binary variable associated with the investment option $k$ of the corridor $i j$, and $k \in \Omega_{2} . f_{i j}^{0}$ is the active power flow in the base network or existing circuits. $f_{i j, k}$ is the active power flow in the $k$ investment option of the expansion circuits. $\bar{f}_{i j}$ is the maximum flow allowed for a circuit in the $i j$ corridor. $\bar{g}$ is the maximum nodal generation vector. $n_{i j}$ is the number of added circuits in the $i j$ corridor. $\bar{n}_{i j}$ is the maximum number of circuits that can be added in the $i j$ corridor. $M$

is a parameter defined a priori of great size, which makes the disjunctive restrictions irrelevant when the variable $y_{i j, k}=0$ (Domínguez, 2017).

In this model, (1) represents the objective function and characterizes traditional planning as a minimum cost problem. The restriction (2) represents the first Kirchhoff law. The restriction (3) allows to establish the limits of capacity in the existing circuits and the (4) in the expansion circuits. Through (5) the generation limits are established and (6) establishes the investment limit. (7) establishes an order of priority among the investment options and eliminates the existing symmetry between these options in the traditional disjunctive transport model. (8) and (9) guarantee that the active power flows are equal in circuits connected in parallel in the same corridor (Garver, 1970; Escobar L, 2018).

\subsection{Cycle formulation}

We use below the basic terminology of graph theory, which will guide us to the definition of the cycle. An unmanaged graph $G$ is a pair $(V, E)$, where $V$ is a finite set and $E$ is a family of (non-ordered) pairs of $V$ elements. The elements of $V$ are called nodes or vertices and the elements of $E$ are called paths or corridors of $G$. Given a corridor between two vertices $i, j \in V$, with $i \neq j$, we denote this corridor for $(i, j)$. Therefore, for a corridor $e=(i, j) \in E, i$ and $j$ are called their final points or vertices. In the same way we say that the $e$ corridor is incident to the vertices $i$ and $j$. Similarly, we say that the vertex $i$ is adjacent to the vertex $j$. The degree of a vertex in a non-directed graph is the number of links or corridors incident to it, which we will denote as $\operatorname{deg}\left(i_{q}\right)$. A path $p$ of length $k$, which joins a vertex $i$ to a vertex $j$, in a graph $G(V, E)$, is a sequence $\left\langle r_{0}, r_{1}, \ldots, r_{k}\right\rangle$ of vertices such that:

$$
i=r_{0}, j=r_{k}
$$


with $\left(r_{m-1}, r_{m}\right) \in E$ for $m=1,2, \ldots, k$. A path is simple if all its vertices are different. In an undirected graph, a path $\left\langle r_{0}, r_{1}, \ldots, r_{k}\right\rangle$ forms a cycle if $r_{0}=r_{k}$ and $r_{1}, r_{2}, \ldots, r_{k}$ are different (Kocuk et al, 2016).

A graph $G^{\prime}=\left(V^{\prime}, E^{\prime}\right)$ is a subgraph of $G=(V, E)$ if $V^{\prime} \subseteq V$ and $E^{\prime} \subseteq E$. Given a set $V^{\prime} \subseteq V$, the subgraph of $G$ induced by $V^{\prime}$ is the graph $G^{\prime}=\left(V^{\prime}, E^{\prime}\right)$ where $E^{\prime}=\left\{(i, j) \in E: i, j \in V^{\prime}\right\}$.

\subsection{Cycle basis}

Let $G=(V, E)$ be an unmanaged graph with $m$ links and $n$ vertices. A cycle of $G$ is a subgraph of $G$. The vector space generated by the incident vectors of cycles is called space of cycles of $G$, which has as dimension:

$$
m-n+\alpha(G),
$$

where $m$ is the number of links or corridors of $G, n$ is the number of nodes or vertices and $\alpha(G)$ is the number of related components of $G$. The set maximal of linearly independent cycles are called cycle basis.

It is important to note that the $G$ links have an assigned weight or value. Therefore, a base of cycles where the sum of the cycle weights is minimal is called minimum cycle basis of $G$. In our case, the $G$ graph is not connected and, through an iterative process, nodes not connected to the system can be added if this allows lower cost solutions. Given that the final system $G^{\prime}=\left(V^{\prime}, E^{\prime}\right)$ obtained is connected but of smaller dimension than $G$, we will denote the dimension of the final space of cycles as:

$$
N=m-n+1,
$$

where $m$ is the number of links in the final solution of $G^{\prime}, n$ is the number of nodes or vertices in the final solution $G^{\prime}$ and $\alpha\left(G^{\prime}\right)=1$ because the The resulting subgraph in the final solution is always connected in the problem of planning the expansion of transmission systems.

\subsection{Solution Methodology}

The methodology used to solve the planning problem is presented below, replacing Kirchhoff's second law with the concept of critical cycles.

A cycle is called a critical cycle if it meets one of the following two conditions:

- It presents circuits with power flows at its maximum capacity, forms cycles with existing and/or added circuits, and the sum of the link weights is minimal.

- It presents additions of circuits in new corridors, forms cycles with existing and/or added circuits, and the sum of the weights of the links is minimal.

Since the critical cycles replace the effect of Kirchhoff's second law, in the planning model, the weights of the links are associated with the bus-angle difference $\left(\theta_{i}-\theta_{j}\right)$ of the power system corridors. If $C$ is a critical system cycle, then:

$$
\sum_{(i, j) \in \mathcal{C}}\left(\theta_{i}-\theta_{j}\right)=\sum_{(i, j) \in \mathcal{C}} w_{i j} f_{i j} x_{i j}=0,
$$

where $C$ is any oriented cycle and $x_{i j}$ is the reactance in the path $(i, j)$. Therefore, the mathematical model for the planning problem of the long-term expansion of transmission systems involving Kirchhoff's second law can be modified using the model given by the equations (1) - (9) plus the minimum cycles given by (10).

Associated with each link $e \in E$, of a critical cycle $C$, there is a value $w_{i j}$ defined as:

$$
w_{i j}=\left\{\begin{array}{l}
1, \quad \text { if the link } i j \in C \text { and has the orientation of } C \\
-1, \quad \text { if the link } i j \in C \text { and has an opposite orientation to the } C \\
0, \quad \text { if the link } i j \notin C
\end{array}\right.
$$

\subsection{Procedure for Generating Critical Cycles}

In an expansion planning problem, given the initial network of the system and the future generation data, future demand and electrical characteristics of the investment options in lines and transformers, the problem is solved using the disjunctive transport model (which does not includes Kirchhoff's second law), and it is verified in the response if circuits appear in their maximum capacity or circuits added in new corridors. The cycle containing these circuits is then determined and is a cycle with minimum sum of weights. These cycles, called critical cycles, are added to the transport model and the process is repeated until no new circuits appear at their upper limit or no new circuits are added in expansion corridors. 
When the critical cycles are formed exclusively by existing corridors, their general form is:

$$
\sum_{(i, j) \in C} w_{i j}\left(\frac{f_{i j}^{0}}{n_{i j}^{0}}\right) x_{i j}=0
$$

As an example of application, in the South-Brazilian system one of the critical cycles assumes the following form:

$$
\left(\frac{f_{12}^{0}}{n_{12}^{0}}\right) x_{12}+\left(\frac{f_{25}^{0}}{n_{25}^{0}}\right) x_{25}+\left(\frac{f_{58}^{0}}{n_{58}^{0}}\right) x_{58}-\left(\frac{f_{78}^{0}}{n_{78}^{0}}\right) x_{78}-\left(\frac{f_{17}^{0}}{n_{17}^{0}}\right) x_{17}=0,
$$

that corresponds to the succession of vertices: $\langle 1,2,5,8,7,1\rangle$. The positive terms correspond to links that have the same orientation of the cycle and the negative ones to the links that have opposite orientation to the cycle.

When critical cycles include circuits in new corridors, the general form of Eq.(10) involves a disjunctive constraint:

$$
\sum_{(i, j) \in C_{1}} w_{i j}\left(\frac{f_{i j}^{0}}{n_{i j}^{0}}\right) x_{i j}+\sum_{(i, j) \in C_{2}} w_{i j} f_{i j, 1} x_{i j} \leq M\left(z-\sum_{(i, j) \in C_{2}} y_{i j, 1}\right), \forall(i, j) \in C
$$

where $C_{1}$ represents the subset of links in the critical cycle $C$, associated with circuits in existing corridors, $C_{2}$ represents the subset of links in the critical cycle $C$, associated with circuits in new brokers, $z$ is the number of links contained in $C_{2}$ to which the binary decision variables $y_{i j, 1}$ are associated.

For the South-Brazilian system, one of the critical cycles involving new corridors corresponds to the succession of vertices:

$$
\langle 5,6,46,19,18,13,8,5\rangle \text {. }
$$

This cycle assumes the following form:

$$
\begin{gathered}
\left\{\left(f_{5-6,1}\right) \cdot x_{5-6}+\left(f_{6-46,1}\right) \cdot x_{6-46}-\left(\frac{f_{19-46}^{0}}{n_{19-46}^{0}}\right) x_{19-46}-\left(\frac{f_{18-19}^{0}}{n_{18-19}^{0}}\right) x_{18-19}-\right. \\
\left.\left(\frac{f_{13-18}^{0}}{n_{13-18}^{0}}\right) x_{13-18}-\left(\frac{f_{8-13}^{0}}{n_{8-13}^{0}}\right) x_{8-13}-\left(\frac{f_{5-8}^{0}}{n_{5-8}^{0}}\right) x_{5-8}\right\} \leq M\left(2-y_{5-6,1}-y_{6-46,1}\right)
\end{gathered}
$$

\section{Results}

The results obtained are presented below, applying the methodology to the South-Brazilian system and the Colombian system of 83 nodes and 155 corridors.

\subsection{South-Brazilian system}

By applying the proposed methodology to the South-Brazilian system, the critical cycles that are presented below are obtained: 
Table 1. Critical cycles South-Brazilian system

\begin{tabular}{|c|c|}
\hline Cycle 1 & $\left(\frac{f_{12}^{0}}{n_{12}^{0}}\right) x_{12}+\left(\frac{f_{25}^{0}}{n_{25}^{0}}\right) x_{25}+\left(\frac{f_{58}^{0}}{n_{58}^{0}}\right) x_{58}-\left(\frac{f_{78}^{0}}{n_{78}^{0}}\right) x_{78}-\left(\frac{f_{17}^{0}}{n_{17}^{0}}\right) x_{17}=0$ \\
\hline Cycle 2 & $\left(\frac{f_{45}^{0}}{n_{45}^{0}}\right) x_{45}+\left(\frac{f_{59}^{0}}{n_{59}^{0}}\right) x_{59}-\left(\frac{f_{49}^{0}}{n_{49}^{0}}\right) x_{49}=0$ \\
\hline Cycle 3 & $\left(\frac{f_{914}^{0}}{n_{914}^{0}}\right) x_{914}+\left(\frac{f_{1418}^{0}}{n_{1418}^{0}}\right) x_{1418}-\left(\frac{f_{1318}^{0}}{n_{1318}^{0}}\right) x_{1318}+\left(\frac{f_{813}^{0}}{n_{813}^{0}}\right) x_{813}-\left(\frac{f_{58}^{0}}{n_{58}^{0}}\right) x_{58}+\left(\frac{f_{59}^{0}}{n_{59}^{0}}\right) x_{59}=0$ \\
\hline Cycle 4a & $\left(f_{56,1}\right) \cdot x_{56}+\left(f_{646,1}\right) \cdot x_{646}-\left(\frac{f_{1946}^{0}}{n_{1946}^{0}}\right) x_{1946}-\left(\frac{f_{1819}^{0}}{n_{1819}^{0}}\right) x_{1819}-\left(\frac{f_{118}^{0}}{n_{1318}^{0}}\right) x_{1318}-$ \\
\hline & $\left(\frac{f_{813}^{0}}{n_{813}^{0}}\right) x_{813}-\left(\frac{f_{58}^{0}}{n_{58}^{0}}\right) x_{58} \leq 100$ \\
\hline Cycle 4b & $\left(f_{56,1}\right) \cdot x_{56}+\left(f_{646,1}\right) \cdot x_{646}-\left(\frac{f_{1946}^{0}}{n_{1946}^{0}}\right) x_{1946}-\left(\frac{f_{1819}^{0}}{n_{1819}^{0}}\right) x_{1819}-\left(\frac{f_{1318}^{0}}{n_{1318}^{0}}\right) x_{1318}-$ \\
\hline & $\left(\frac{f_{813}^{0}}{n_{813}^{0}}\right) x_{813}-\left(\frac{f_{58}^{0}}{n_{58}^{0}}\right) x_{58} \geq-10$ \\
\hline Cycle 5 & $\left(\frac{f_{1422}^{0}}{n_{1422}^{0}}\right) x_{1422}+\left(\frac{f_{2226}^{0}}{n_{2226}^{0}}\right) x_{2226}-\left(\frac{f_{1426}^{0}}{n_{1426}^{0}}\right) x_{1426}=0$ \\
\hline Cycle 6 & $\left(\frac{f_{1617}^{0}}{n_{1617}^{0}}\right) x_{1617}+\left(\frac{f_{1719}^{0}}{n_{1719}^{0}}\right) x_{1719}+\left(\frac{f_{1946}^{0}}{n_{1946}^{0}}\right) x_{1946}-\left(\frac{f_{1646}^{0}}{n_{1646}^{0}}\right) x_{1646}=0$ \\
\hline Cycle 7 & $\left(\frac{f_{1318}^{0}}{n_{1318}^{0}}\right) x_{1318}+\left(\frac{f_{1820}^{0}}{n_{1820}^{0}}\right) x_{1820}-\left(\frac{f_{1320}^{0}}{n_{1320}^{0}}\right) x_{1320}=0$ \\
\hline Cycle 8 & $\left(\frac{f_{1819}^{0}}{n_{1819}^{0}}\right) x_{1819}+\left(\frac{f_{1921}^{0}}{n_{1921}^{0}}\right) x_{1921}-\left(\frac{f_{2021}^{0}}{n_{2021}^{0}}\right) x_{2021}-\left(\frac{f_{1820}^{0}}{n_{1820}^{0}}\right) x_{1820}=0$ \\
\hline Cycle 9 & $\left(\frac{f_{1426}^{0}}{n_{1426}^{0}}\right) x_{1426}+\left(\frac{f_{2627}^{0}}{n_{2627}^{0}}\right) x_{2627}+\left(\frac{f_{2738}^{0}}{n_{2738}^{0}}\right) x_{2738}+\left(\frac{f_{3842}^{0}}{n_{3842}^{0}}\right) x_{3842}+\left(\frac{f_{4243}^{0}}{n_{4243}^{0}}\right) x_{4243}-$ \\
\hline & $\left(\frac{f_{3243}^{0}}{n_{3243}^{0}}\right) x_{3243}-\left(\frac{f_{1932}^{0}}{n_{1932}^{0}}\right) x_{1932}-\left(\frac{f_{1819}^{0}}{n_{1819}^{0}}\right) x_{1819}-\left(\frac{f_{1418}^{0}}{n_{1418}^{0}}\right) x_{1418}=0$ \\
\hline Cycle 10 & $\left(\frac{f_{2434}^{0}}{n_{2434}^{0}}\right) x_{2434}-\left(\frac{f_{3334}^{0}}{n_{3334}^{0}}\right) x_{3334}-\left(\frac{f_{2433}^{0}}{n_{2433}^{0}}\right) x_{2433}=0$ \\
\hline Cycle 11 & $\begin{array}{l}\left(\frac{f_{2023}^{0}}{n_{2023}^{0}}\right) x_{2023}+\left(\frac{f_{2324}^{0}}{n_{2324}^{0}}\right) x_{2324}+\left(\frac{f_{2434}^{0}}{n_{2434}^{0}}\right) x_{2434}+\left(\frac{f_{3435}^{0}}{n_{3435}^{0}}\right) x_{3435}+\left(\frac{f_{3538}^{0}}{n_{3538}^{0}}\right) x_{3538}- \\
\left(\frac{f_{2738}^{0}}{n_{2738}^{0}}\right) x_{2738}-\left(\frac{f_{2627}^{0}}{n_{2627}^{0}}\right) x_{2627}-\left(\frac{f_{1426}^{0}}{n_{1426}^{0}}\right) x_{1426}+\left(\frac{f_{1418}^{0}}{n_{1418}^{0}}\right) x_{1418}+\left(\frac{f_{1820}^{0}}{n_{1820}^{0}}\right) x_{1820}=0\end{array}$ \\
\hline
\end{tabular}

Cycle 4 presented in the previous table involves two new corridors, and corresponds to a disjunctive constraint that occurs in two parts cycle $4 \mathrm{a}$ and cycle $4 \mathrm{~b}$. When the methodology is applied, 11 cycles are obtained and adding them to the disjunctive transport model allows obtaining the best known solution for this system of US $\$ 72,870$ million dollars, with an execution time of $0.48 \mathrm{~s}$ and 591.56 ticks. It is important to note that the computation times with this methodology is much more efficient than using the traditional disjunctive model (including the second Kirchhoff law), in effect, with this model we obtain an execution time of $0.86 \mathrm{~s}$ and 1188.97 ticks. The ticks are a computer-independent measure of how much algorithmic work is required to obtain a provable optimum, independently of the computer on which it is run on.

\subsection{Colombian System}

By applying the proposed methodology to the Colombian system, the critical cycles that are presented below are obtained: 
Table 2. Critical cycles Colombian system

\begin{tabular}{|l|l|}
\hline \hline Cycle $1^{*}$ & $\langle 52,88,43,39,68,86,19,82,55,57,56,54,47,52\rangle$ \\
\hline Cycle $2^{*}$ & $\langle 57,81,56,57\rangle$ \\
\hline Cycle 3 & $\langle 25,28,29,25\rangle$ \\
\hline Cycle 4 & $\langle 14,31,60,14\rangle$ \\
\hline Cycle 5 & $\langle 14,18,20,13,14\rangle$ \\
\hline Cycle 6 & $\langle 2,83,9,69,70,34,4,2\rangle$ \\
\hline Cycle 7 & $\langle 15,18,20,15\rangle$ \\
\hline Cycle 8 & $\langle 19,61,68,86,19\rangle$ \\
\hline Cycle 9 & $\langle 61,68,37,61\rangle$ \\
\hline Cycle 10 & $\langle 24,75,12,76,17,23,24\rangle$ \\
\hline Cycle 11 & $\langle 27,35,44,27\rangle$ \\
\hline Cycle 12 & $\langle 45,50,54,45\rangle$ \\
\hline Cycle 13 & $\langle 59,67,68,61,19,66,69,9,8,59\rangle$ \\
\hline Cycle 14 & $\langle 8,59,1,8\rangle$ \\
\hline Cycle 15 & $\langle 1,3,71,1\rangle$ \\
\hline Cycle 16 & $\langle 55,62,82,55\rangle$ \\
\hline
\end{tabular}

\begin{tabular}{|l|l|}
\hline \hline Cycle 17 & $\langle 66,69,60,14,18,66\rangle$ \\
\hline Cycle 18 & $\langle 9,69,70,34,4,2,9\rangle$ \\
\hline Cycle 19 & $\langle 31,32,34,31\rangle$ \\
\hline Cycle 20 & $\langle 16,18,21,16\rangle$ \\
\hline Cycle 21 & $\langle 31,34,33,72,31\rangle$ \\
\hline Cycle 22 & $\langle 31,33,72,31\rangle$ \\
\hline Cycle 23 & $\langle 31,60,69,70,34,32,31\rangle$ \\
\hline Cycle 24 & $\langle 18,20,13,23,16,18\rangle$ \\
\hline Cycle 25 & $\langle 18,66,19,58,18\rangle$ \\
\hline Cycle 26 & $\langle 19,22,21,18,58,19\rangle$ \\
\hline Cycle 27 & $\langle 17,23,24,15,17\rangle$ \\
\hline Cycle 29 & $\langle 1,8,71,3,1\rangle$ \\
\hline Ciclo 30 & $\langle 19,58,18,22,19\rangle$ \\
\hline Cycle 31 & $\langle 27,64,29,27\rangle$ \\
\hline Cycle 32 & $\langle 27,44,80,27\rangle$ \\
\hline Cycle 33 & $\langle 26,27,28,26\rangle$ \\
\hline
\end{tabular}

\begin{tabular}{|l|l|}
\hline \hline Cycle 34 & $\langle 27,29,25,28,27\rangle$ \\
\hline Cycle 35 & $\langle 64,65,30,72,73,74,64\rangle$ \\
\hline Cycle 36 & $\langle 4,34,33,72,30,64,27,35,36,4\rangle$ \\
\hline Cycle 37 & $\langle 8,9,77,79,87,8\rangle$ \\
\hline Cycle 38 & $\langle 23,24,15,18,14,13,23\rangle$ \\
\hline Cycle 39 & $\langle 6,10,78,7,90,3,6\rangle$ \\
\hline Cycle 40 & $\langle 45,81,56,54,45\rangle$ \\
\hline Cycle 41 & $\langle 64,74,73,72,30,64\rangle$ \\
\hline Cycle 42 & $\langle 72,73,62,60,31,72\rangle$ \\
\hline Cycle 43 & $\langle 19,82,62,60,69,66,19\rangle$ \\
\hline Cycle 44 & $\langle 82,85,83,9,69,60,62,82\rangle$ \\
\hline Cycle 45 & $\langle 1,93,92,11,1\rangle$ \\
\hline Cycle 46 & $\langle 9,83,85,91,90,3,71,8,9\rangle$ \\
\hline Cycle 47 & $\langle 29,31,72,30,64,29\rangle$ \\
\hline Cycle 48 & $\langle 85,91,92,93,1,8,9,83,85\rangle$ \\
\hline Cycle 49* & $\langle 27,89,74,64,27\rangle$ \\
\hline Cycle 50 & $\langle 4,5,6,3,71,8,9,2,4\rangle$ \\
\hline
\end{tabular}

Cycles 1, 2 and 49 presented in the previous table involve new corridors, and correspond to a disjunctive constraint. With the proposed methodology applied to the Colombian electricity system (using the disjunctive transport model), $50 \mathrm{critical}$ cycles were obtained, which allow finding the best known solution for this system of US 562,417 million dollars. The execution time was $62.79 \mathrm{~s}$ with $96484.45 \mathrm{~s}$. As in the previous system, the methodology proposed in this work allows to obtain better results in computation times, that is, with the traditional disjunctive model the same optimal solution is obtained but with execution time of $99.25 \mathrm{~s}$ with 147153.02 ticks, which shows the effectiveness of the proposal with cycles.

\section{Conclusion}

The DC model considered the ideal model to solve the planning problem of the transmission expansion can be replaced by the disjunctive transport model plus the set of critical system cycles. In medium and high complexity systems, this sample is a promising technique to reduce computation times.

\section{Acknowledgements}

This work was supported by the Planning Group in Power Electric Systems and GEDNOL group research at the Technological University of Pereira - Colombia, and the Brazilian institutions CAPES, CNPq (Grant No. 142150/2015-0) and Sao Paulo Research Foundation-FAPESP (Grant No. 2015/21972-6).

\section{References}

Escobar, A. (2010). Modelos usados en el planeamiento de la expansión a largo plazo de sistemas de transmisión de energía eléctrica. Universidad Tecnológica de Pereira.

Domínguez, A. (2014). Metodología de solución para el planeamiento de la transmisión considerando incertidumbre en la demanda y propuestas de diferentes conductores. Revista EIA. Colombia. (Vol. 11, pp. 99-112). http://dx.doi.org/10.14508/reia.2014.11.21.99-112

Escobar, L. (2016). Long term transmission expansion planning considering generation-demand scenarios and HVDC 
lines. IEEE PES Transmission \& Distribution Conference and Exposition-Latin America (PES T\&D-LA). http://dx.doi.org/10.1109/TDC-LA.2016.7805617

Escobar, L. (2018). Specialized models for the long-term transmission network expansion planning problem. Ph.D thesis. Universidade Estadual Paulista (UNESP). SP Brasil.

Domínguez, A. (2017). Planeamiento multietapa a largo plazo de redes de transmisión considerando alternativas HVDC, pérdidas y contingencias. Tesis Doctoral en Ingeniería, Universidad Tecnológica de Pereira.

Garver, L. (1970). Transmission Network Estimation Using Linear Programming. Power Apparatus and Systems, IEEE Transactions. (Vol. 89, pp. 1688-1697). http://dx.doi.org/10.1109/TPAS.1970.292825

Escobar, A. (2002). Planeamiento dinámico de la expansión de sistemas de transmisión usando algoritmos combinatoriales. Tesis de Maestría, Universidad Tecnológica de Pereira.

Kocuk, B. (2016). A Cycle-Based Formulation and Valid Inequalities for DC Power Transmission Problems with Switching. Operations Research. (Vol. 64, pp. 922-938). http://dx.doi.org/10.1287/opre.2015.1471

\section{Copyrights}

Copyright for this article is retained by the author(s), with first publication rights granted to the journal.

This is an open-access article distributed under the terms and conditions of the Creative Commons Attribution license (http://creativecommons.org/licenses/by/3.0/). 